\title{
Open lower limb fractures in the UK trauma system: A multicentre prospective audit of
}

\section{current practice.}

\begin{abstract}
Introduction

Open fractures represent limb-threatening and life-changing injuries. Clear standards define how patients with these injuries should be managed in the UK. The study of open fractures is, therefore, a key measurable example of major trauma management as a whole. This study was conducted to characterise the demographic, assessment and treatment of patients sustaining lower limb open fractures across UK Regional Trauma Networks.
\end{abstract}

\section{Methods}

A prospective, multicentre, audit was conducted according to a prespecified protocol against the relevant British Orthopaedic Association (BOA) \& British Association of Plastic, Reconstructive \& Aesthetic Surgeons (BAPRAS) standards for Trauma. All UK hospitals treating adults with open fractures were eligible to take part in the study. Patients included were injured during a six month collection period at each site.

\section{Results}

3 Major Trauma Centres (MTCs) and 5 Trauma Units (TUs) were enrolled, with data collected by 24 collaborators. 239 patients were included, 11 had bilateral open fractures. There were 38 patient datasets collected from TUs and 201 from MTCs. Patients were predominantly male with high energy injuries.

$31.3 \%$ of patients from MTCs were transferred in from another facility. Antibiotics were given to $41.7 \%$ of patients within 1 hour. $74.4 \%$ of limbs with open fractures had a splint applied in the 
emergency department. $85.8 \%$ of patients had a documented orthoplastics plan. $41.7 \%$ of patients with a high energy injury had their wound debrided within 12 hours.

\section{Discussion}

$42.3 \%$ of patients with open fractures in our cohort were managed at some point in a TU, indicating triage was required within the trauma network. Due to sampling, we may be underestimating the number of patients passing through TUs, however, we have demonstrated that this cohort exists. These patients are under-represented in many other studies and registries such as the Trauma Audit Research Network (TARN) due to the funding model for data collection that privileges data collection in MTCs.

\section{Conclusion}

This study gives extensive new insight into the demographics and management of patients with open lower limb fractures in the UK, demonstrating a widespread involvement of TUs and consistent deviation from national standards.

\section{Highlights}

1. A prospective, multicentre, audit including 239 patients with open fractures from 3 Major Trauma Centres and 5 Trauma Units in the UK.

2. $31.3 \%$ of patients from Major Trauma Centres were transferred in from another facility.

3. $42.3 \%$ of patients with open fractures were managed at some point in a Trauma Unit.

4. BOAST/BAPRAS Open Fracture standards are poorly adhered to across the study population.

5. Trauma unit patients are excluded in many other studies and registries due to the model for data collection that privileges data collection in MTCs. 


\section{Main Text}

\section{Introduction}

Open fractures represent limb-threatening and life-changing injuries which can affect any individual in society. ${ }^{[1]}$ The British Orthopaedic Association (BOA) and British Association of Plastic, Reconstructive and Aesthetic Surgeons (BAPRAS) publish guidance for the management of open fractures which have become national standards for all patients injured in the UK. ${ }^{[2]}$ These standards comprehensively cover all aspects of patient care from the assessment on scene and triage, to in-hospital management processes and timings for all open fractures of the long bones, hindfoot and midfoot. Further guidance on these fractures and for open fractures of other bones can be found from the National Institute for Health and Care Excellence (NICE) national guidance for open fractures. ${ }^{[3]}$

Adherence to these standards has been shown to improve outcomes for patients. ${ }^{[4-5]}$ Delays to transfer or treatment of these fractures are independent predictors of poor outcomes such as infection ${ }^{[6-7]}$, with one study finding a $3 \%$ increase in infection with each one hour delay to

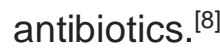

The new UK open fracture standards coincide neatly with the introduction of the Regional Trauma Networks (RTN) in 2012. This system was established in a hub and spoke format, each with one or more specialist Major Trauma Centres (MTCs) and peripheral Trauma Units (TUs). These network systems significantly improve outcomes for severely injured patients. ${ }^{[9]}$

According to BOAST standards, patients sustaining open long bone fractures should be transferred to MTCs - either triaged pre-hospital or early transfer from a TU. Open fractures require early interventions from specialist teams, ${ }^{[10]}$ are relatively common, easily diagnosed, and should be treated according to clear standards. They, therefore, can be used as a unique 
tracer condition to assess major trauma systems across the UK and their implementation of timely, effective care. RTNs still vary in their performance and pathways for traumatically injured patients, in particular with little known about the management of open fractures in TUs

This study aimed to characterise the demographics, assessment and treatment of patients sustaining lower limb open fractures across the UK RTNs and audit these findings against the BOAST national standard. Additionally, the study aimed to establish whether there are underreported numbers of patients managed in TUs that should have been transferred to MTCs earlier or a silent cohort that were not transferred at all. These would therefore not be captured in previous studies or registries such as the Trauma Audit Research Network (TARN) due to the way in which data collection is funded and organised primarily in MTCs. We hypothesise that these may predominantly include low energy open fractures in frail elderly patients - previously shown to receive poor triage, slow interventions and suffer worse outcomes - comparable to outcomes seen in neck of femur fractures. ${ }^{[11-12]}$

\section{Patients and Methods}

\section{Study design}

A prospective, multicentre, audit was conducted according to a prespecified protocol against the agreed BOAST standards.

\section{Reporting}

This study is reported according to Strengthening the Reporting of Observational Studies in Epidemiology (STROBE) guidelines. ${ }^{[13]}$

\section{Setting}


UK hospitals treating patients with open fractures were eligible to take part in the study. Patients included were injured during a six month collection period at each site. Each patient was followed up for 30 days from their presentation. The first site opened in May 2017 and the final site closed in March 2018.

\section{Participants}

All adults presenting with open lower limb fractures were eligible for inclusion. Collaborators prospectively identified patients from on-call lists, trauma meetings, and operating lists. Patients were followed up using electronic patient records and medical notes during and following their admission.

\section{Variables}

Data were collected on patient demographics: age, sex, Rockwood Frailty Score. ${ }^{[14]}$ Data relating to the injury included Gustilo and Anderson grade ${ }^{[15]}$, contamination, Glasgow Coma Scale (GCS), Injury Severity Score (ISS), mechanism of injury, and the presence of polytrauma defined as two or more severe injuries in at least two areas of the body. Admissions were captured as direct to that hospital or a transfer from elsewhere. Data on management related to orthoplastics plans, tetanus toxoid, antibiotics, splintage, wound excision, definitive and temporary stabilisation and soft tissue coverage. Data on outcomes related to return to theatre, wound infection (any depth), ITU admission, amputation, and death.

\section{Data sources}

Collaborators collected data from electronic patient records and medical notes for each variable. Obligatory data fields were used to aid data completeness.

\section{Bias}


To address selection bias, eligible patients were included in a prospective and consecutive fashion.

\section{Study size}

The study aimed to collect data on all patients presenting to each centre during the six month period.

\section{Quantitative variables}

Quantitative variables were categorised as either normally distributed or not normally distributed using the Shapiro-Wilk test and were reported using appropriate descriptive statistics for each category. Groupings of quantitative variables were defined a priori and the data were collected in these groupings.

\section{Collaborative Network}

24 trainee collaborators were recruited using social media, mailing lists, and the British Orthopaedic Network Environment. ${ }^{[16]}$ All collaborators were supervised by a consultant Trauma and Orthopaedic surgeon. This observational study collected only routine and non-identifiable data. Research Ethics Committee approval was not required under the assessment of the National Health Service Health Research Authority decision tool. ${ }^{[17]}$ Collaborators were required to gain local research and audit department approval from their respective institutions. This established model of collaborative research has previously been described in detail elsewhere. ${ }^{[18]}$ Collaborators are recognised in accordance with national guidelines. ${ }^{[19]}$

\section{Database}

A bespoke data collection form was designed by $\mathrm{OB}, \mathrm{HC}$, and $\mathrm{XG}$. Data were entered onto a database on the REDCap (Research Electronic Data Capture) online system. ${ }^{[20]}$ Data were 
stored securely on REDCap servers at the Nuffield Department of Orthopaedics, Rheumatology, and Musculoskeletal Science (NDORMS) at the University of Oxford. Entries were checked centrally and where updates or clarifications were required, these were updated by local investigators.

\section{Statistical methods}

Continuous data were described using mean and standard deviation (normally distributed) or median and interquartile range (not normally distributed). Categorical data were described using frequencies. No imputation of missing data was performed. Collaborators were required to attest that 30-day follow up was completed for all patients involved in the study to minimise loss to follow up. The chi-squared test was used to compare differences among categorical variables. The Mann-Whitney test was used to compare continuous variables (not normally distributed) between categorical groups. Statistical significance was ascribed when the $p$-value was $<0.05$. Statistical analyses were performed using Prism (GraphPad, San Diego, CA, USA).

\section{Results}

Hospitals

3 MTCs (Oxford, Bristol, The Royal London) and 5 TUs (Swansea, Reading, Lewisham, Stoke Mandeville, Cramlington) were enrolled into this study and data were collected by 24 collaborators (table 1). At the time of data collection, the Welsh trauma network was not established. Morriston Hospital (Swansea) is considered as a TU here, though it is a large hospital with specialist services e.g. plastic surgery. Stoke Mandeville hospital in Aylesbury also has plastic surgical input from its burns centre.

\section{Patients}


239 patients with open fractures were included in the study. 11 patients had bilateral open fractures. The median age was 45.8 years, 158 (66.1\%) were male and 81 (33.9\%) were female. Median Rockwood frailty score was 2 (table 3). There were 38 patients included from TUs and 201 from MTCs. No patients were included twice having been seen at a TU and then transferred to an MTC. The median age was significantly lower in males with open fractures than women $(P<0.0001)$ (fig 1). Mechanism of injury varied significantly by gender $(P<0.0001)$ (figs 2-3). There was no association between the mechanism of injury and the admission type (direct/transfer) or type of hospital (MTC/TU).

There was no association between Gustilo and Anderson grade and the type of hospital at which the patient was assessed (MTC/TU). There was no association between frailty and admission type (direct/transfer) or the type of hospital at which the patient was assessed (MTC/TU). The distribution of age was not different across categories of admission type (direct/transfer) or the type of hospital at which the patient was assessed.

\section{Injury}

$61(25.5 \%)$ patients sustained injuries as part of a polytrauma (two or more severe injuries in at least two areas of the body). Glasgow Coma Scale (GCS) score was 15 in $91.6 \%$ and median ISS was 9 (table 2). Table 3 describes the location of the open fracture(s), Gustilo and Anderson grade and presence of wound contamination.

\section{Management}

$101 / 239$ (42.3\%) patients with open fractures in our cohort were managed at some point in a TU. 63/201 patients (31.3\%) included from the 3 MTCs were transferred in from another centre. $83 \%$ of these transfers were directly from an emergency department (ED) shortly after 
diagnosing an open fracture. The remaining $17 \%$ were transferred after surgical intervention in a TU (table 3).

Tetanus toxoid was given to $73.4 \%$ of patients and antibiotics were given to $94.1 \%$ of patients (table 3). Time to first antibiotic dose is shown in figure 4. Co-amoxiclav was the most commonly used drug and was given to $65.2 \%$. Antibiotics were given within 1 hour of arrival to hospital to $41.7 \%$ and within 2 hours to $67.6 \%$. Tables $3-4$ contain further information on antibiotic use.

$74.4 \%$ of limbs with open fractures had a splint applied in the Emergency Department. 85.8\% of patients had a documented combined Orthoplastics plan (table 4).

Temporary stabilisation was performed in $56.9 \%$ and definitive stabilisation was performed in 85.8\% (table 3, figs 5-6). Definitive stabilisation was performed within 72 hours in $76.1 \%$ (table 3).

Wound excision was performed in $74.9 \%$. Time to wound excision is outlined in tables $3-4$. $41.7 \%$ of patients with a high energy injury had their wound excised within 12 hours (fig 7). 9 patients (11 limbs) had heavily contaminated (marine/ agricultural/ sewerage) wounds. Data on time to wound excision were available for $6 / 9$ patients. 1 had their wound excised in $<2$ hours, 4 in $12-<24$ hours, 1 in $36-<48$ hours.

Definitive soft tissue coverage was performed in $72.4 \%$ (tables $2-3$, fig 8 ). This was performed by an orthopaedic surgeon in $51.7 \%$ and a plastic surgeon in $47.2 \%$. A consultant performed the soft tissue coverage in $65.7 \%$ (table 3 ). 


\section{Outcome}

$6.9 \%$ had a wound infection (any depth) following their operation. $14.2 \%$ of patients returned to theatre during this admission for revision surgery following definitive fixation and soft-tissue coverage, the reasons for return are outlined in table 3. $7.8 \%$ of injured lower limbs were amputated and time to amputation is outlined in table $4.12 .1 \%$ were admitted to ITU as part of their treatment. Length of ITU stay is outlined in table 3. 3\% of patients died whilst in hospital. When outcomes were divided into treating hospitals, wound infections were most common in TUs (12.5\%), whilst ITU admissions and returns to theatre were commonest in those patients directly transferred to MTCs. There were no amputations or deaths in patients treated at any point in TUs (Table 5).

$34.4 \%$ of open fractures were of the severe Gustilo and Anderson 3b or 3c types. Among this sub-group 9.3\% had a wound infection (any depth), 20.9\% returned to theatre during the admission for revision surgery, $18.6 \%$ were treated with amputation, $19.8 \%$ were admitted to ITU as part of their treatment, and $6.7 \%$ died whilst in hospital.

All patients were followed up using hospital electronic patient records and medical notes for 30 days following their injury. Patients were not telephoned and GPs were not contacted to capture outcome data that did not lead to readmission or outpatient contact with the hospital following discharge.

The dataset is not complete for all variables. Tables report the data completeness for each variable by giving the number of patients, limbs, or fractures the data relates to. We have reported where collaborators have entered 'unknown' for a variable.

\section{Discussion}


This multicentre, prospective collaborative study provides a broad snapshot of current practice for open fracture demographics, management and outcomes across several regional trauma networks in the UK. Surprisingly little is currently known on the true demographics and outcomes for this important group of injuries, which represent a key indicator of trauma care as a whole.

There is a bimodal distribution of younger males and older females sustaining open fractures as seen in other studies. ${ }^{[12]}$ Low energy injuries were predominantly seen in elderly females, whereas high energy injuries were predominantly seen in young males. We find little definitive evidence of a 'silent cohort' of frail, elderly, low energy fractures managed in TUs. Of note, this study has considered 19 patients from Morriston Hospital (Swansea) as being from a TU as this is accurate for the time of data collection before the establishment of the Welsh Trauma Network. This centre does, however, have a number of specialists and equipment not available in other TUs in England such as combined orthoplastics care.

63/201 patients (31.3\%) included from the 3 MTCs were transferred in from another centre. $83 \%$ of these were early transfers from one Emergency Department to another suggesting that this triage could potentially have been done on the scene and the patient taken to the appropriate centre. The remainder had orthopaedic surgery in the TU before being transferred, which contravenes BOAST standards unless that TU can provide comprehensive orthoplastic care. When these 63 transferred patients are added to the 38 patients included from TUs, this represents 101/239 (42.3\%) patients with open fractures in our cohort managed at some point in a TU.

The ratio of TUs to MTCs in the UK is greater than reflected in this study. Due to sampling, we may be under-estimating the number of patients passing through TUs, however, we have 
demonstrated that this cohort exists. These patients are excluded in many other studies and registries such as TARN due to the way that data collection predominantly is funded and takes place in MTCs. These patients should still be managed according to BOAST/BAPRAS standards. It may be that further evidence from TUs is required over longer time periods to further identify this cohort.

Despite clear BOAST national standards on the management of open fractures, both MTCs and TUs are failing to consistently meet targets. Notably on time taken to give antibiotics, perform wound excision, and have a formal orthoplastics plan. Adherence to these targets is known to reduce the length of stay and improve outcomes. ${ }^{[6-7,10]}$ Our data on time to antibiotics is based on the time from admission to hospital. The BOAST/BAPRAS open fractures standard recommends administration $<1 \mathrm{~h}$ from injury. By collecting data from hospital records, we were not reliably able to establish the time of injury and may not have recorded antibiotics given prehospital. The administration of tetanus toxoid in $73.4 \%$ is also of interest although not in the BOAST standards. Tetanus immunoglobulin may also be advisable in cases of heavy contamination.

This study also establishes that temporary management of the fracture is predominantly in cast or external fixator and permanent fixation mainly a plate or intramedullary nail. We also establish that the time taken to perform these operations does not universally meet national standards.

Rates of infection, return to theatre and amputation reflect those seen in the recent literature. ${ }^{[12,21]}$ We also provide new insight into reasons for return to theatre in 33 patients, namely further washout/debridement, soft tissue revision or revision of skeletal stabilisation in equal proportions. These numbers are based on the 30-day follow up period and may increase over 
time. There are clearly significant delays in deciding to perform amputations, which must be addressed, but further research is required into the management pathways for this complex and emotive decision. It is of interest to report that TUs have a higher infection rate in open fractures. There were, however, small numbers of patients treated only in TUs, therefore imprecision in estimates precluded valid statistical analysis on differences in outcome with further potential confounding due to patient triage.

Some elements of the BOAST standards could not be audited accurately with our data collection tool as we felt higher value should be placed on accurate reporting of certain key parameters. Standards not audited were: frequency of neurovascular examinations, treatment of arterial injuries, use of CT and angiograms, the existence of any 'mini-washouts' before theatre, photographs of the injury in the notes, use of fresh instruments in theatre after draping, decision processes for definitive stabilization or amputation including involvement of the family and wider MDT. We did not audit whether these patients had data submitted to TARN or whether patients were given suitable information about recovery and rehabilitation.

This study investigates a large cohort of lower-limb open fractures sustained within a short time frame across a number of different centres, providing information on a more substantial proportion of the UK population than many other series published. Data were collected on multiple aspects of patient demographic, management and outcome, many of which are not to our knowledge reported by other large studies.

A recent paper using the TARN database focussed on tibial fractures of Gustilo and Anderson grade $3 \mathrm{~B}$ and above managed only in MTCs. This reports similar timings to antibiotics and wound debridement and highlights a potential tendency to delayed management for the elderly. ${ }^{[12]}$ This paper, while high in patient numbers, suffers a high percentage of missing data 
(44\% on Abx timing). A further longitudinal study on motorcyclists with open fractures using TARN data has looked at adherence to some key BOAST open fracture standards with large patient numbers. ${ }^{[22]}$ The TARN database does not report on all of the BOAST standards included in this study or the breadth of data such as frailty score, tetanus toxoid, types of procedure performed, soft tissue cover and reasons for returning to theatre.

Targets for antibiotics, debridement, stabilisation and coverage have also been variably missed in other studies performed in the UK. ${ }^{[12,21,23-24]} \mathrm{A}$ combined ortho/plastics approach has also been identified as variably met in 33-92\%. ${ }^{[12,23-24]}$ Further studies conclude however that despite targets being missed, there remains a lack of evidence regarding the actual clinical impact of timely debridement or delivery of antibiotics. ${ }^{[12,25-27]}$

This study should be considered in view of potential limitations. Due to the questionnaire design and investigator follow up, there are low numbers of incomplete data compared to other studies performed, which improves reliability. However, there may be a number of patients presenting during the time period that were not captured by this snapshot. This may represent a selection bias if for example low severity injuries which are not managed in MTCs or with brief hospital stays may be more likely missed. This snapshot style study does not follow up patients beyond the 30 day follow up which was asked of collaborators. This means we are unable to capture readmissions to hospital for chronic infection or further procedures. We did not collect data on all cases in the UK during the recruitment period. Our results may therefore not reflect the standards being followed in other trusts.

\section{Conclusion}

Whilst patients from MTCs represent the vast majority of our submitted data, nearly half of all included patients were managed in a TU at some point in their journey, 
contraveningBOAST/BAPRAS standards. It is clear that there is significant variation across the UK in choice of antibiotics and methods of stabilisation, fixation or coverage. Open fractures represent a wide range of injuries and act as a unique litmus test for the trauma networks as a whole, with clear evidence that national standards are not being uniformly met. Further research is needed to establish why and to ascertain the impact these findings may have on outcomes. Collecting accurate and thorough data across multiple TUs still presents a significant difficulty.

\section{Acknowledgements - none}

Data Access - Additional data are available upon request to the corresponding author References

1. Giannoudis P.V., Harwood PJ, Kontakis G, Allami M, Macdonald D, Kay SP, et al. Long-term quality of life in trauma patients following the full spectrum of tibial injury (fasciotomy, closed fracture, grade IIIB/IIIC open fracture and amputation). Injury 2009;40:213-9.

2. British Orthopaedic Association Standards for Trauma. December 2017. Accessed 13 Jun 2020. [Available from: https://www.boa.ac.uk/resources/boast-4-pdf.html].

3. National Institute for Health and Care Excellence. Fractures (complex): assessment and management. 2017. Accessed 13 Jun 2020. [Available at: https://www.nice.org.uk/guidance/ng37]

4. Stammers J, Williams D, Hunter Jet al. The impact of trauma centre designation on open tibial fracture management. Ann R Coll Surg Engl 2013; 95: 184-187.

5. Wordsworth M, Lawton G, Nathwani D, Pearse M, Naique S, Dodds A, et al. Improving the care of patients with severe open fractures of the tibia: the effect of the introduction of Major Trauma Networks and national guidelines. Bone Joint J 2016;98:420-4.

6. D'Alleyrand JC, Manson TT, Dancy L et al. Is time to flap coverage of open tibial fractures an independent predictor of flap-related complications? J Orthop Trauma 2014; 28: 288-293. 
7. Pollak AN, Jones AL, Castillo RC, Bosse MJ, MacKenzie EJ, LEAP Study Group. The relationship between time to surgical debridement and incidence of infection after open highenergy lower extremity trauma. J Bone Joint Surg Am 2010;92:7-15.

8. Hull PD, Johnson SC, Stephen DJG, Kreder HJ, Jenkinson RJ. Delayed debridement of severe open fractures is associated with a higher rate of deep infection. Bone Jointt J 2014;96 B:379-84. doi:10.1302/0301-620X.96B3.

9. Moran CG, Lecky F, Bouamra O. Changing the System - Major Trauma Patients and Their Outcomes in the NHS (England) 2008-17. EClinicalMedicine. 2018;0(0)

10. Nayagam S, Graham K, Pearse M, Nanchahal J. Reconstructive surgery in limbs: the case for the orthoplastic approach. Ann Plast Surg 2011;66:6-8.

11. Chang DC, Bass RR, Cornwell EE, Mackenzie EJ. Undertriage of elderly trauma patients to statedesignated trauma centers. Arch Surg 2008;143:776-81.

12. Young K, Aquilina A, Chesser TJS, Costa ML, Hettiaratchy S, Kelly MB, Moran CG, Pallister I, Woodford M; Open tibial fractures in major trauma centres: A national prospective cohort study of current practice. MTC-22. Injury. 2019 Feb;50(2):497-502. doi: 10.1016/j.injury.2018.10.028. Epub 2018 Oct 29.

13. Von Elm E, Altman DG, Egger M, Pocock SJ, Gotzsche PC, Vandenbroucke JP. The Strengthening the Reporting of Observational Studies in Epidemiology (STROBE) Statement: guidelines for reporting observational studies.

14. Rockwood K, Song X, MacKnight C, et al. A global clinical measure of fitness and frailty in elderly people. CMAJ. 2005;173(5):489-495. doi:10.1503/cmaj.050051.

15. Gustilo RB, Anderson JT. Prevention of infection in the treatment of one thousand and twenty-five open fractures of long bones: Retrospective and prospective analyses. J Bone Joint Surg Am. 1976;58:453-8.

16. British Orthopaedic Network Environment. Accessed 13 Jun 2020. [Available at: https://www.bone.ac.uk/].

17. NHS Health Research Authority Ethics Decision Tool. Accessed 13 Jun 2020. [Available at: http://www.hra-decisiontools.org.uk/research] 
18. Bhangu A, Kolias AG, Pinkney T et al. Surgical research collaborative in the UK. Lancet 2013; 382: 1091-2.

19. Recognising contributions to work in research collaboratives: Guidelines for standardising reporting of authorship in collaborative research.National Research Collaborative \& Association of Surgeons in Training Collaborative Consensus Group. Int J Surg. 2018 Apr;52:355-360. Doi: 9.1016/j.ijsu.2017.12.019. Epub 2017 Dec 29.

20. Harris PA, Taylor R, Thielke R, Payne J, Gonzalez N, Conde JG, Research electronic data capture (REDCap) - A metadata-driven methodology and workflow process for providing translational research informatics support, J Biomed Inform. 2009 Apr;42(2):377-81.

21. Hendrickson S, Wall R, Manley O, Gibson W, Toher D, Wallis K, Ward J, Wallace D, Lamyman M, Giblin A-V, Wright T, Khan U.Time to Initial Debridement and wound Excision (TIDE) in severe open tibial fractures and related clinical outcome: A multi-centre study. Injury, 2018.

22. Hay-David A, Stacey T, Pallister I, Trauma Audit and Research Network Research Committee UioM. Motorcyclists and pillion passengers with open lower-limb fractures: a study using TARN data 2007-2014. Ann R Coll Surg Engl. 2018;100(3):203-8.

23. Rymer B, Dimovska EOF, Chou DTS, Choa R, Davis B, Huq S. A representative assessment of the management of open fractures of the lower limb within UK orthoplastic centres: A two-centre audit of compliance with national standards. Injury. 2017;48(10):2266-9.

24. Yarrow J, Rahman S, Marsden N, Pallister I, Hemington-Gorse S. Management of open lower limb injuries in South West England and Wales. Ann R Coll Surg Engl. 2015;97(1):35-9.

25. Lack WD, Karunakar MA, Angerame MR, Seymour RB, Sims S, Kellam JF, et al. Type III open tibia fractures: immediate antibiotic prophylaxis minimizes infection. J Orthop Trauma 2015;29:16.

26. Melvin JS, Dombroski DG, Torbert JT, Kovach SJ, Esterhai JL, Mehta S. Open tibial shaft fractures: I. Evaluation and initial wound management. J Am Acad Orthop Surg 2010;18:10-9.

27. Hendrickson SA, Donovan R, Harries L, Wright TC, Whitehouse MR, Khan U. Time to intravenous antibiotic administration (TIbiA) in severe open tibial fractures: Impact of change to national guidance. Injury. 2020;51(4):1086-1090. doi:10.1016/j.injury.2020.03.005 


\section{Figure legends}

- Figure 1 - Ages of patients with open fractures of each gender (Line at median, bars show $\left.95 \% \mathrm{Cl}, \mathrm{P}<0.0001^{* \star * *}\right)$.

- Figure 2 - Patient age and gender distributions

- Figure 3 - Mechanism of injury by gender

- Figure 4 - Time to first antibiotic dose

- Figure 5 - Type of temporary stabilisation used

- Figure 6 - Type of definitive stabilisation used

- Figure 7 - Time to wound excision in 96 high energy fractures

- Figure 8 - Time to definitive soft tissue coverage

\section{Table legends}

- Table 1 - Hospitals included

- Table 2 - Descriptive statistics of continuous variables

- Table 3 - Descriptive statistics of categorical variables

- Table 4 - Adherence to BOAST/BAPRAS open fractures guideline

- Table 5 - Outcomes across different patient journeys 